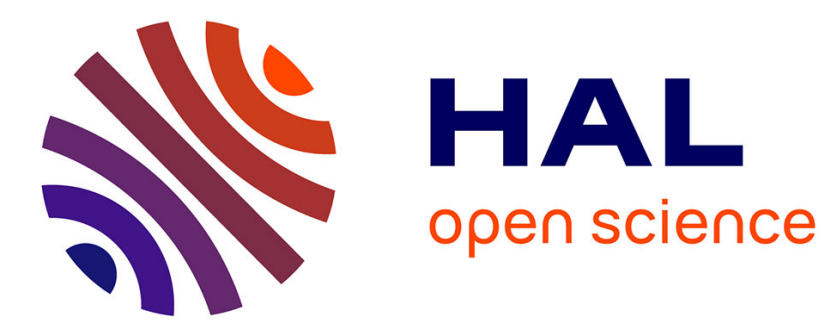

\title{
Gestion du risque et de l'aléatoire par les pêcheurs martiniquais
}

\author{
Isabelle Dubost
}

\section{To cite this version:}

Isabelle Dubost. Gestion du risque et de l'aléatoire par les pêcheurs martiniquais. G. Blanchet, B. Gobert, J. A. Guérédrat. La pêche aux Antilles, Martinique, Guadeloupe, 2002. hal-01655123

HAL Id: hal-01655123

https://hal.science/hal-01655123

Submitted on 11 Dec 2017

HAL is a multi-disciplinary open access archive for the deposit and dissemination of scientific research documents, whether they are published or not. The documents may come from teaching and research institutions in France or abroad, or from public or private research centers.
L'archive ouverte pluridisciplinaire HAL, est destinée au dépôt et à la diffusion de documents scientifiques de niveau recherche, publiés ou non, émanant des établissements d'enseignement et de recherche français ou étrangers, des laboratoires publics ou privés. 


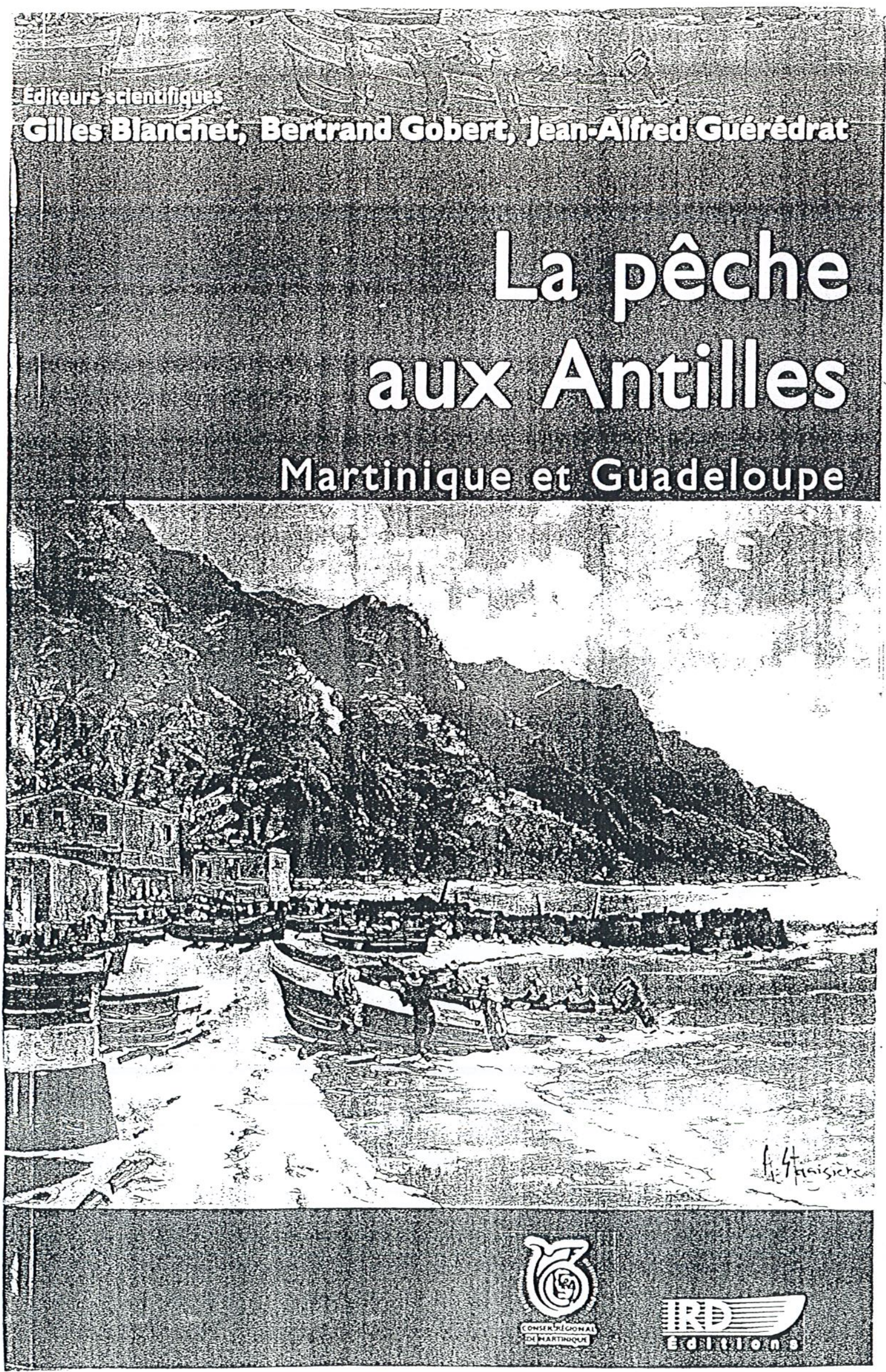




\title{
La pêche aux Antilles (Martinique et Guadeloupe)
}

\author{
Éditeurs scientifiques
}

Gilles BlanCHET, Bertrand Gobert, Jean-Alfred GuÉRÉDrat

Ouvrage publié avec le concours

du Conseil régional de la Martinique

IRD Éditions

INSTITUT DE RECHERCHE POUR LE DÉVELOPPEMENT

Paris, 2002 


\section{Gestion du risque et de l'aléatoire par les pêcheurs martiniquais}

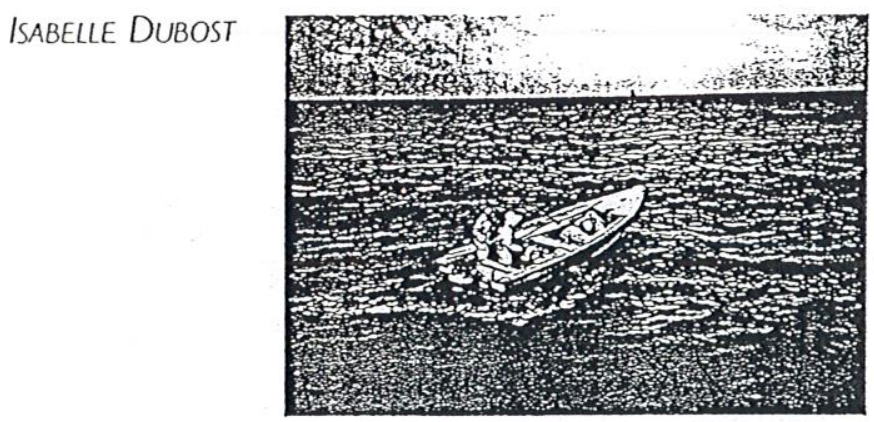

O Hremerla Guiliou

\section{Introduction}

Lobjectif de tout pêcheur est de réussir : réussir sa journée par des prises abondantes en toute sécurité, réussir à atteindre un statut de «bon » pêcheur. Pour cela, il fait des choix selon son savoir-faire, ses connaissances et selon ses engins de pêche, afin de gérer au mieux les aléas inhérents à son métier, ceux liés à une ressource renouvelable et à un environnement «inconnu » et dangereux quill convient de maitriser autant que possible. En effet, la mer est un espace de dérive à conquérir, le lieu d'une recherche à la fois éperdue et rationnelle de la ressource afin de se lapproprier. Tout pêcheur est donc tributaire des contraintes quill apprend à gérer en se fondant sur son apprentissage et sur son expériencé. devenant, à son tour, créatif.

Au-delà des dangers et des aléas que subit chaque travailleur de la mer. le pêcheur martiniquais est confronté à des contraintes propres à son univers social particulier. Nous essaierons de montrer ici que ces risques et ces aléas ne sont pas seulement liés à l'exercice du métier, mais sont aussi fonction des relations que chaque pêcheur doit entretenir avec les autres et dont il doit en même temps se méfier. en marquant bien la limite entre l'espace privé qui est le sien et l'espace public auquel il se rattache. Ainsi, sans se dérober au regard des « autres », dans un univers de grande proximité et de surveillance mutuelle. pour être efficace un pêcheur doit s'arranger de ces contraintes, les dépasser lorsque cela est nécessaire et en gérer les conséquences. afin d'éviter des conflits potentiels. 
Lo péche oux Antilles

(Martinique, Guodeloupe)

\section{Ne pas échapper au regard de l'autre...}

\section{De la proximité au risque de la promiscuité}

Les pêcheurs se sont installés sur le littoral. sur la bande des cinquante pas géométriques' ou dans la mangrove avoisinante qu'ils ont défrichée. faute de pouvoir s'approprier les terres ${ }^{2}$ de lintérieur. Cette occupation d'espaces limités et peu accueillants s'est souvent faite sans acte de propriété mais n'en conduit pas moins les pêcheurs à transmettre leurs biens immobiliers à leurs descendants. Ainsi ce sont de véritables lignées familiales qui occupent ces terres. La mobilité n'étant pas de mise, on y vit et on y travaille en «vase clos».

Ce mode d'habiter entraine une forte socialisation, voire parfois une promiscuité, qui aboutit à un contrôle social de tous les instants.

Un jeu permanent d'observation et de démonstration en résulte qui ne laisse rien à l'ignorance des voisins, des membres de la famille ou des amis. Tout se voit, se regarde, tout s'observe avec attention car tout passe par la reconnaissance de l'autre, de ses actes, de sa vie, de sa possession de biens... Aussi, seul celui qui se dérobe au regard est mal perçu car cela suggère quil a quelque chose à cacher. Cependant, un code implicite conduit chacun à trouver une intimité et surtout à la préserver, ce qui est tout un art dans cette société où la socialisation est si forte et le paraître si important. Regarder l'autre est une manière de s'en rapprocher et de créer du lien social. Cela suppose également d'être regardé, ce qui induit une mise en scène de sa vie afin de donner à voir, mais uniquement ce qui est souhaitable.

Prenons l'exemple de Grand-Rivière, bourgade du nond de lîle, lovée au pied de falaises. Sept quartiers se partagent une bande de terre d'un kilomètre : «La Lave », « Bagasse », « Perriolat », «L'aut'Bord », « Bellevue », « Case Maillart », « Le Bourg». Les maisons se jouxtent, ne laissant qu'un étroit passage entre elles. appelé «couloir», tandis que des ruelles délimitent un espace plus large où se situent des boutiques d'alimentation et quelques restaurants. Ces «couloirs», d'une largeur à peine plus importante que la carrure d'un individu, ne sont pas coinsidérés comme privés puisque des non-résidents peuvent y circuler. Il convient cependant de saluer toute personne rencontrée pour signifier la présence et le respect. Le manquement à cet usage est considéré comme une marque d'impolitesse. Toute rencontre dans l'espace commun ne peut être anonyme, elle doit être marquée par la reconnaissance de l'autre. Elle passe par la parole. l'indifférence n'étant pas tolérée.

\footnotetext{
' Zone correspondant à $81.20 \mathrm{~m}$, appartenant à l'État et gérée par les Domaines. Les demières législations interdisant l'occupation des cinquante pas géométriques posent un véritable dilemme aux autorité qui devraient obliger les pêcheurs à quitter les lieux. Des conflits surgissent régulièrement à ce propos.

$2 \dot{A}$ la Martinique. l'appropriation de la terre demeure une problématique complexe: la majorité des terres à vocation agricole (vastes plaines plantées en canne à sucre ou en banane) appartient encore aux békés (descendants des colons français) qui représentent environ $2 \%$ de la population. II existe une grande parcellisation des terres restantes appartenant à de petits planteurs. constituées par des lopins parfois en indivision.
} 
II riest guère aisé, dans ces conditions, de se cacher des voisins. En effet, tout se sait des activités des uns et des autres. "II n'y a pas d'intimité » disait une femme de Grand-Rivière, «on s'entend d'une maison à une autre». Se disputer entre époux, réprimander un enfant, regarder la télévision ou accueillir un étranger sont autant de faits qui n'échappent pas à autrui. Le moindre déplacement est repéré et enregistré. La circulation est d'ailleurs très codifiée, les déplacements sont fonctionnels, l'errance n'existe pas. Pour les femmes, cela se réduit aux visites au marché, aux boutiques et à l'église, tandis que les hommes circulent entre la maison. le site où est entreposé le matériel de pêche, le mouillage du canot et différents lieux de rencontre:

C'est là que les hommes s'échangent les informations sur la vie collective, individuelle et sur la pêche. Chaque soir, les pêcheurs se réunissent dans les cafés, sur les places ou au bord de mer pour discuter, jouer aux dominos ou à la pétanque. Le marché et la rivière sont les rares lieux de mixité. En plus des activités économiques, ménagères ${ }^{3}$ ou ludiques qui leur sont liées, ils sont propices à l'échange. C'est ici que se consolident les liens interpersonnels, les femmes quittant pour un temps leur univers domestique.

Sur le plan professionnel, l'observation réciproque est de mise. Elle sert d'émulation car connaitre la situation de l'autre permet de mesurer sa propre réussite et de laméliorer. Mais au-delà d'une concurrence, elle est aussi le support d'une surveillance.

\section{De l'observation à la surveillance}

La pêche est un métier fondé sur des facteurs aléatoires comme la présence des bancs de poissons, les conditions météorologiques... Elle est marquée par une très forte concurrence, surtout lorsque les ressources halieutiques se raréfient comme c'est le cas à la Martinique4, d'où l'importance d'être bien informé. C'est pourquoi, une attention très vive est apportée aux paroles, aux réactions et aux comportements des uns et des autres, tant à terre qu'en mer, afin de déceler les atouts et les difficultés rencontrés. Mais le secret est aussi inhérent à ce métier. À terre, les discussions entre pêcheurs portent souvent sur le métier : «on se raconte toutes sortes d'histoires, mais la conversation la plus importante, c'est la pêche et les courses de yols. II n'y a jamais de bagarres mais la raison du plus fort est toujours la meilleure ». En fait, les récits de pêche ne révèlent jamais des actes

\footnotetext{
${ }^{3}$ Nombreuses sont les femmes qui lavent leur linge à la rivière (BOUGEROt. 1984) ou le rincent après un lavage en machine. car leau a une valeur de purification. C'est pourquoi des bains. appelés ben démoroj sont pris le 31 décembre pour préparer la nouvelle année. Les canots subissent le méme traitement avec une préparation à base de plantes (DuBOSt. 1996). On pêche aussi les zabiton. écrevisses, et les titiri. juvéniles despèces différentes deau douce et d'eaụ salée. $\dot{A}$ Grand-Rivière. la rivière tient une place majeure dans la vie collective.

1 Les espèces de poisson à proximité des côtes sont péchées depuis longtemps avec les mémes engins tandis que les espèces qui vivent au large ou en profondeur ont fait lobjet dune moindre exploitation

s Canot de péche en bois ou en plastique utilisé pour des régates. La carène a été transformée. ce qui a donné des canots spécifiques pour les régates depuis plusieurs années (DUBOSt. 1993).
} 
Lopéche oux Antilles

(Morcinique. Guadeloupe)

précis concernant une technique particulière, une zone de pêche riche en poissons. les quantités pêchées... On peut apprendre qu'un coup de senne ${ }^{6}$ se prépare et qu'un banc de poissons se profile au large des côtes. que les prix de vente sur les marchés se maintiennent... Ne sont divulguées que les informations générales, faciles à obtenir par l'observation qui relèvent d'un simple savoir-faire collectif. Par contre, tout ce qui appartient au savoir-faire individuel, à des connaissances acquises au fil des années par l'expérience n'est pas à partager. Chaque individu garde jalousement ses secrets.

Il convient alors de s'observer entre pêcheurs pour découvrir les bonnes zones de pêche ou l'épav' - épave - qui amène tant de poissons ou, au contraire, de se prémunir dune telle observation. Pour déjouer les rencontres inopportunes sur un lieu de pêche, it faut user de «malice » et de «ruse». Ainsi, on pêche en «reculant » petit à petit avec le kannot ${ }^{8}$ - canot -. pour revenir ensuite sur le lieu initial. Sur une épov durant la saison de pêche à miklon' - miquelon -, il est préférable de se cacher pour rester seul et ainsi travailler à volonté. Pour cela, on ôte le ciré, pour ne pas être visible de loin ; on retire le capot du moteur en simulant une réparation ou le plein d'essence : on arrête le moteur car la fumée se voit au loin... On peut aussi simuler d'autres occupations: se cacher sous un banc du kannot, ralentir la cadence, manger son casse-croûte, ranger son matériel à bord. Pour subtiliser l'épov, on la coule sous le kannot ou on la met à bord, alors que ce n'est pas admis dans les codes. La meilleure « ruse » est de simuler un départ afin que le concurrent s'en aille : à la place de l'épav cachée à bord, une bovée est mouillée. donnant ainsi lillusion d'un casier déposé. De l'huile de foie de rétjen'o - requin -. est déposée sur l'eau, formant un glasi - glacis - à la surface, ce qui permet de retrouver la zone de pêche. Enfin. pour éloigner les oiseaux qui marquent la présence d'une épov, on leur donne à manger plus loin. Puisqu'il est possible de retrouver un banc de poissons le lendemain en suivant le courant, un pêcheur use de stratégie pour ne pas être escorté en partant à l'opposé. Alors,

\footnotetext{
6 Technique de pêche collective dencerclement diun banc de poissons avec un filet halé de la plage par une vingtaine d'individus (DUBOST, 1996).

'L'épor - épave - ou le bwo - bois - est un objet flottant sour lequel se réfugient de nombreux poissons pour profiter de l'ombre comme les dorad - dorade (Corphæna hippurus). les bous - bourses - (Balistidæ + Monacanthidæ). les toza - thazands - (Scomberomorus brosiliensis + S. covolla + Aconthocybium solondn). les ton - thons - (Scombridæ)... (DuBOst. 1996).

${ }^{8}$ Canot en bois ou en polyester. II existe des yol. canot à charpente première. et des gonmyé. pirogue monoxyle. de moins en moins utilisés.

- C'est la pêche la plus valorisée et la plus valorisante. Dune part. elle fait appel à toutes les qualités physiques (force. résistance...) et cognitives diun pêcheur. d'autre part elle est la plus rentable. De fin décembre à début juillet, jusquà 50 milles des côtes. les pêcheurs mettent en œuvre différentes techniques comme la pêche au volon (Exocetidæ) au filet. à la ligne ou au kali (sorte d'épuisette), la pèche à la ligne de traine de surface et à la dérive. Sa dénomination fait référence à léloignement des côtes (DUBOST. 1996).

${ }^{10}$ (Corcharhinus spp. + Sphygina spp. + Sphyrna zygæno)
} 
les concurrents, par lassitude, détournent leur route. C'est, en effet, une règle que de suivre systématiquement un pêcheur réputé pour le surprendre et connaître ses prises".

\section{De la dissimulation à l'ostentation}

Observer ses confrères ou user de «ruse » est donc tout un art. II s'agit d'un jeu ambigu entre dissimulation et ostentation, entre discrétion et provocation. Ainsi, un «bon » pêcheur est apte à déjouer l'observation de ses collègues en dissimulant sa pratique par la «ruse», tout en exhibant sa réussite par des signes extérieurs'".

En effet, un pêcheur doit montrer sa réussite afin de susciter l'envie et ainsi être estimé pour le statut que lui confèrent ses compétences. Sa «ruse » fait partie de son savoir-faire. À la Guadeloupe, hors du milieu de pêcheurs, BOUGEROL (1997 : 15-21) montre que cette pratique est de mise : «si le plus souvent il s'agit de tromper le regard de l'autre, il arrive aussi, que de façon plus singulière, certains jouent avec la surveillance et la suscitent par des comportements provocateurs» [...] «cette conduite de provocation est pensée comme un mauvais tour à l'endroit de celui qui surveille » [...] « cette mise en scène qui se nomme "donner une occupation" a pour but de punir ». Pour les pêcheurs martiniquais, ce qui est exhibé s'adresse d'abord à un voisin ou un collègue puis à la collectivité. Cependant, personne n'est dupe de cette mise en scène collective : chacun peut quantifier les possessions des autres.

En réalité, la réussite n'est pas le résultat d'un pur dépassement de soi dans le but d'un contentement individuel. Elle n'a de sens que comparée à la situation des autres, ce que R PRICE (1964: 99) confirme pour Petite Anse, village de pêcheurs du sud de liile : «Le succès en matière de pêche n'est que rarement évalué en termes absolus; l'unique étalon de réussite reste la comparaison avec la prise du voisin ». La réussite est avant tout sociale, dans la confrontation à l'autre et doit donc s'exhiber.

\footnotetext{
"Les pêcheurs estiment les prises selon le tempérament et l'humeur des membres de l'équipage. Ainsi, tel patron tacitume est de bonne humeur lorsqu'il a bien pêché. tel autre silencieux devient bavard... Si le kannot. au retour. a sa ligne de flottaison au ras de leau, cela signifie que la joumée a été fructueuse. Si la tête d'un poisson dépasse du kannot. cela montre que grands et nombreux sont les poissons. En mer. lorsquiun pécheur écope de l'eau claire. cela indique une mauvaise pêche. sinon leau aurait été souillée de sang.

12 Lagrandissement diune maison. lachat d'un nouveau moteur très puissant. la construction diun nouveau canot plus spacieux... sont autant de signes extérieurs de richesse quiun individu montre avec ostentation. tandis quil garde secret ses prises. Aux Saintes (Guadeloupe). jai pu observer less mémes tactiques de dissimulation: un pécheur. réputé pour la péche à la traine. donnait rendez-vous au mareyeur en mer pour lui vendre son produit afin que ses confrères ne puissent estimer ses quantités pêchées. ce qui n'empêchait pas ces derniers de tenter des approches. Par contre, il exhibait son canot neuf et ses deux moteurs de $150 \mathrm{ch}$.
} 
Lo pêche oux Antilles

(Martinique. Guadeloupe)

Détourner le regard de l'autre tout comme se montrer nécessitent donc une grande imagination en inventant des mises en scène et des stratégies. Ce système correspond bien à ce que GOFFMAN (1973:23) définit comme la « totalité de lactivité d'une personne donnée. dans une occasion donnée. pour influencer dune certaine façon un des autres participants».

$\dot{A}$ Grand-Rivière, on dit : «c'est chacun pour soi et Dieu pour la compagnie, chacun se débrouille »! C'est la loi de la concurrence. Pourtant, ni les codes qui réglementent l'accès à la ressource, ni le jeu de la ruse ne peuvent empêcher les conflits qui naîssent de cette compétition entre pêcheurs. Cette attitude généralisée riest pas üniquement professionnelle, liée à la concurrence. Elle sinscrit dans un contexté où limage de soi garantit le statut d'un individu dans une société très hiérarchisée qui valorise la réussite individuelle. Mais celle-ci ne prend toute sa dimension que si elle est entérinée collectivement. Cela passe par une reconnaissance. Observer, être observé permet toujours de mesurer sa situation par rapport aux autres, ce qui induit une parfaite connaissance des rôles et places des uns et des autres. Ainsi, ce qu'impliquent cette observation et cette surveillance est un contrôle social.

\section{Regard et convoitise : un univers conflictuel}

\section{Statut social}

Être pêcheur ne suffit pas, il faut être le meilleur. Durant toute sa carrière, chaque individu va mettre tout en œuvre pour y arriver. Ainsi, il va affiner son savoir et son savoir-faire technique, en développant ses qualités et ses compétences par sa propre expérience et en côtoyant les «Anciens». Est un «bon » pêcheur celui qui ramène de grosses prises régulièrement, ce qui lui assure des revenus convenables et un bon statut social. Sa réussite est donc à la fois technique, économique et sociale.

La réussite entraîne une dynamique de compétition. Par exemple, les jeunes de Grand-Rivière accèdent ensemble aux lieux de pêche tout en se défiant Suite à l'observation intervient la mise en œuvre de stratégies. Comme l'écrit G. SIMMEL (Rééd. 1995: 76). la concurrence induit un « immense effet de socialisation » par la nécessité de se rapprocher de son adversaire pour apprécier ses méthodes. Cependant, une restriction est à faire. Certes, la concurrence « oblige le concurrent, qui voit quil a un rival - et qui bien souvent n'entrerait pas en concurrence sans cela-.à aller au-devant et à se rapprocher de celui qu'on cherche à séduire, à se lier à lui, à étudier ses forces et ses faiblesses et à s'y adapter, à chercher toutes les passerelles qui pourraient relier sa propre personne et son propre travail au sien, ou à les établir ». Mais les pêcheurs gardent une grande distance malgré tout. 
En effet, la concurrence peut très rapidement déboucher sur des conflits extrêmement violents si elle n'est pas contenue. Le lien social se tisse mais peut très vite se délier.

- Effectivement, une réussite nuit dans la mesure où elle se fait au détriment d'autres personnes. Elle génère alors de l'inégalité : de bonnes pêches, des trophées réguliers lors de régates ou tout élément de richesse marque une différenciation alors «que les pêcheurs sont censés avoir les mêmes chances, car avec le même matériel, ils devraient pêcher pareil ». L'individu ainsi privilégié se démarque de ses confrères et compromet l'idéal d'une égalité de statuts Sa conduite est jugée « anormale » et suscite la jalouzi - jalousie.

\section{La jalouzi}

Ce sentiment de désir envers celui qui possède plus, n’est jamais exprimé verbalement en face à face, mais il est facile de déterminer qui peut devenir jalou jaloux.

Comme le dit G. Simmel (Rééd., 1995 : 69), le jaloux pense avoir un droit, ici celui d'acquérir le même statut et les mêmes qualités que son confrère chanceux, ce qui le différencie de l'envieux. Par conséquent, « La jalousie est déterminée [...] par le fait que nous ne pouvons posséder un bien justement parce quil est dans la main de l'autre, et qu'il nous reviendrait aussitôt que cet obstacle serait levé : les sentiments de l'envieux tournent toujours autour de l'objet possédé, ceux du jaloux autour de celui qui le possède » (SIMMEL rééd., 1995 : 66). Un troisième phénomène se glisse entre les deux, le dépit considéré comme «le désir envieux d'un objet, non pas parce qu'il est particulièrement désirable pour le sujet, mais seulement parce que l'autre le possède » (ibid. : 67).

À la Martinique, la jolouzi se situe entre les sentiments de jalousie et d'envie car ce qui est convoité n'est pas tant l'objet (un puissant moteur de yole, par exemple) que ce quill représente : une place respectable dans le groupe. ce à quoi tout individu aspire et considère comme un droit. Ce qui suscite la jolouzi est le désir de jouir d'un avantage égal. L'enjeu ici est bien celui de l'égalité. L'insupportable est de pas obtenir la même chose ou la même qualité qu'un autre individu, de ne pas être son égal.

La jalouzi est perçue comme collective : les «autres» sont des jolous. Celui qui s'écarte de la norme égalitaire prend le risque d'être désigné par tous comme déviant et, de fait, devenir marginalisé. L'excès doit être désigné par la collectivité, même si, bien souvent, la sanction est individuelle. Celle-ci est implicitement soutenue par tous. Là encore, la pression sociale joue à plein par le biais de l'observation. Les jolous sont obligatairement des méchan - méchants - qui vont user de stratégies sorcellaires pour évincer leur rival.

II s'avère que la jolouzi ne suscite pas seulement un désir dimitation chez les pêcheurs. comme BOUGerol (1997: 15) a pu lobserver à la Guadeloupe dans d’autres circonstances, mais aussi un désir de destruction : il faut contrecarrer celui qui réussit. Ce qui est convoité est le résultat et non les moyens. Cela peut se tra. duire par une incapacité réelle à se dépasser. Comme l'exprime si bien un pêcheur: 
Lo pèche oux Antilles

(Marcinique. Guadeloupe)

«la jolouzi sinstalle [dès lors qu'existe] la réussite du moi. C'est vouloir être comme le voisin : je travaille. je n'y arrive pas, l'autre réussit. Au lieu de trouver le moyen d'être comme lui. je le détruis ». La conséquence d'un tel principe est qu'un pêcheur en situation d'échec (mauvaises prises, perte de matériel...) ne remettra pas en doute ses capacités et son savoir-faire, ne cherchera pas à imiter l'autre car la cause de (ses) tels déboires est associée à des actes néfastes.

\section{La sorcellerie comme principe de régulation sociale}

Ce contexte de rivalité fait que le conflit est omniprésent : «entre les hommes. on se déchire. on se tire dans les pattes ». Le conflit éclate ou reste latent avant de s'exprimer ún jour ou l'autre. II demeure cependant l'expression d'une socialisation. Comme le dit Simmel (Rééd., 1995 : 19-20), « ce sont les causes du conflit. la haine et l'envie, la misère et la convoitise, qui sont véritablement l'élément de dissociation. Une fois que le conflit a éclaté pour l'une de ces raisons, il est en fait un mouvement de protection. contre le dualisme qui sépare. et une voie qui mènera à une sorte d'unité, quelle qu'elle soit, même si elle passe par la destruction de lune des parties » [...] «En lui-même, le conflit est déjà la résolution des tensions entre les contraires ».

La jolouzi fomente le mal qui mène à la persécution et à la destruction (DUBOst, 1995). $\grave{A}$ une forme de violence correspond une autre forme de violence : une action «méchante » entraine une action de représailles. Effectivement, le jolou ne se contente pas d'être un simple observateur déçu. il intente des actions pour interférer directement sur la situation enviée. Pour cela, il utilise un arsenal rituel afin de régler cette situation inégalitaire à ses yeux. La sorcellerie et la protection rituelle interviennent alors comme des principes de régulation sociale pour ramener

- les individus à des statuts équivalents. On peut reprendre C. BOUGerol (1977:25) qui cite G. Foster : « dans toute société, les personnes utilisent des formes culturelles symboliques et non symboliques dont la fonction est de neutraliser, d'atténuer ou de contrôler, de quelque manière, les dangers qui découlent de l'envie, et surtout de la crainte de l'envie ».

La sorcellerie règle ou accentue le conflit mais exclut tout compromis. Sa finalité est de mettre un terme au désordre' ${ }^{13}$ ou, au contraire, de favoriser la chans'4. Elle sert à ramener un pêcheur trop chanceux au même rang que ses collègues. Celui-ci voit peser sur lui un regard collectif de suspicion : il est impossible quili soit le seul responsable de sa réussite. Circule alors la rumeur selon laquelle il aurait profité du pouvoir d'un kenbwazè - sorcier -. qui lui aurait fourni tous les objets nécessaires pour favoriser sa production, ou bien encore il est accusé d"avoir passé un pacte avec le djob - diable -, angajmon - engagement - qui requiert une vie en échange. L'individu ainsi visé n'a plus quiune seule solution : se protéger, sinon les « autres» se chargeront de lui nuire.

$$
\vdots .
$$

13 Pèche insuffisante. déboires familiaux...

14 Dans son acception créole. celle-ci ne se définit pas comme le fruit du destin. mais bien plutôt comme le résultat d'un travail rituel qui permet d'accéder à la réussite par de bonnes prises régulières. en minimisant les risques liés à l'environnement tout en se protégeant des «autres». 
Dans cette perspective, le mal's ainsi "envoyé » nuit au pêcheur en tant que professionnel mais aussi en tant qu'individu. c'est-à-dire lhomme dans son être physique, moral et surtout dans sa condition sociale. La force morale va être détruite, le caractère fragilisé. caractérisé par une perte de désir. d'aptitude à prendre des décisions et à gérer son existence. Tout comme FAVRET-SAADA (1977: 335) l'a décrit, l'individu ainsi « attaqué » est «atteint dans sa force, c'est-à-dire dans sa capacité de survie. de reproduction [...] et dans sa production », dans «sa - force vitale ». La seule réponse possible à la sorcellerie est de se protéger par un système global qui inclut des rites, le suivi du calendrier religieux, des fêtes et des interdits, système qui riest pas uniquement lié à la pêche mais qui touche à toutes les sphères de la vie privée.

\section{Un ordre social restauré : l'égalité retrouvée}

La réussite est à la fois désirée et crainte. C'est un souhait et une nécessité pour tout un chacun. Pourtant les moyens employés, tout comme ses effets inquiètent. En effet, la réussite déstabilise lordre social par son principe inégalitaire : elle introduit une hiérarchie entre individus qui perdent leur égalité de statuts. Celui qui réussit trop bien est soupçonné de sorcellerie tandis que celui qui échoue dans son entreprise est considéré comme un perdant qui subit des actes de sorcellerie et qui aurait besoin, à son tour, d'une telle aide ou d'engager une action rituelle personnelle. Ainsi autant la réussite que l'échec entraînent une situation inégalitaire et il n'est pas «normal» qu'un pêcheur réussisse moins bien ou mieux qu'un autre.

La jalouzi est le sentiment suscité par cette différence de résultats. Par son expression, elle montre où se situe le déséquilibre. En effet, le jolou montre du doigt l'inégalité et entreprend des actions pour rétablir l'ordre, c'est-à-dire renouer avec l'égalité, refoulant la sorcellerie. Et l'inégalité est source de conflit, surtout lorsqu'il s'agit d'une désignation de sorcellerie : «l'accusation de sorcellerie est bien un conflit, car elle tend à la rupture ou à la révision d'une relation sociale» (AuGÉ, 1975 : 96).

La sorcellerie est, en fait, un intermédiaire actif au cœur d'un conflit pour le résoudre ou pour l'aviver en en supprimant ou en exagérant la cause. Pour un temps. la sorcellerie marginalise lindividu atteint, qui est montré du doigt par la suspicion. ou souffre de ses effets. Mais dès que la crise est résolue. celui-ci retrouve son statut antérieur. Ainsi un très bon pêcheur deviendra un simple pêcheur, voire un pêcheur malchanceux. un pêcheur dans l'échec retrouvera sa productivité. L'égalité entre pêcheurs est retrouvée et l'individu est alors ramené au sein du groupe en même temps que le contrôle social est restauré.

Même si les pêcheurs réprouvent le recours à la sorcellerie. ils la considèrent comme un principe constitutif de leur univers social et y font appel lorsque cela est nécessaire

La sorcellerie a donc une fonction de compensation et de régulation sociale. Elle intervient en cas de désordre. en le neutralisant par un autre désordre. Lun 
La péche aux Antilles

(Martinique. Guadeloupe)

annulant l'autre. lordre est alors restauré : « le pouvoir de la sorcellerie se présente partout comme instituant un ordre en rapport avec l'ordre social » (AUGÉ, 1975: 91). L'inégalité est détruite. L'ordre retrouvé est alors celui de l'égalité qui «prend la forme diun strict contrôle social» (BONNIOL, 1980 : 292). Seule le maintien d'un espace privé permet de se protéger de ce contrôle social et de ses conséquences.

\section{Espace public-espace privé : un équilibre fragile}

\section{Espace privé}

La $k a z$ - case - est le symbole même d'une répartition entre espace public et espace privé. Elle a une double configuration : centrée sur elle-même autour de la pièce principale et de la cuisine attenante, et ouverte sur l'extérieur avec la terrasse où se déroule la vie quotidienne. Des règles codifient l'accès des maisons car ces lieux sont strictement réservés à l'usage familial. Quiconque pénètre dans une maison doit émettre un appel, tototo, pour signifier sa venue et demander I'hospitalité.

Le centre de la maison est un lieu privé que l'on préserve absolument, alors que les abords et la terrasse sont les zones intermédiaires de circulation. Les fenêtres en verre s'ouvrent sur les passages entre maisons, elles sont souvent sans rideaux et obligatoirement ouvertes pour laisser passer l'air. Elles donnent à voir ce qui se passe à l'intérieur alors, qu'autrefois, les jalousies ne dévoilaient rien grâce aux lattes en bois orientables. Au cœur d'une maison, les chambres ne sont jamais montrées, c'est l'espace privé ultime. À la rigueur le salon, mais de préférence la cuisine adjacente, sont les lieux privilégiés pour recevoir une visite. Le soir, tout s'inverse. Dès la tombée du jour ou après le repas, toutes les ouvertures sont fermées, la famille se replie sur elle-même, se cloître dans son espace. Seuls les hommes sortent.

Cette habitude de se préserver est ancienne car, d'après BONNIOL (1982: 73), «les premiers esclaves ont, selon Du Tertre, leurs cases closes comme des boites enfumées par un feu permanent». II poursuit, «nous touchons là un trait qui a persisté jusqu'à la case actuelle qu'on continue à calfeutrer soigneusement la nuit pour se protéger contre les coups de lune et les esprits malfaisants».

Pour la Guadeloupe. BenOít (1989: 140) dit que «le souci de protection de l'espace habité $[. .$.$] renvoie à une organisation en "coquilles" ». Cette organisation$ est une garantie : "la case est une des "coquilles" de lhabitat de par son organisation et non pas simplement pàr ses murs, tout comme la peau protège de par la présence de godko (garde-corps, amulettes) et non en tant que telle. Ce sont ces plus-values culturelles qui assurent l'efficacité de la protection » (ibid. : 149). En effet, « les différentes "coquilles" sont des barrières qui protègent la case et ses 
Lo péche aux Antilles

(Martinigue. Guodeloupe)

habitants des agressions extérieures. La première coquille comprend les limites de la parcelle et les plantes magiques du jardin. La seconde correspond à la zone soigneusement balayée autour de la case. La troisième est constituée par les murs de la case sur lesquels peuvent être ajoutées des protections » (ibid. : 154).

La kaz demeure le seul territoire individuel et familial qui tout à la fois protège des peurs nocturnes et des esprits malfaisants et protège des « autres » car il permet de se dérober à leur surveillance, bref au contrôle social. Dans la vie quotidienne, des attitudes permettent également de maintenir une barrière entre soi et les « autres».

\section{Espace public}

Les relations de voisinage illustrent bien aussi cette nécessaire séparation pour protéger l'intimité individuelle ou familiale. Elles sont caractérisées par la discrétion et le retrait. Dans un quartier, tous s'interpellent d'une fenêtre à l'autre pour. demander des nouvelles, des discussions s'engagent parfois, mais la distance demeure la norme. Chaque personne reste chez elle : «on ne va pas par plaisir chez les gens ». Les voisins pénètrent rarement à l'intérieur de la maison, ils sont reçus sur la terrasse, à l'entrée ou sur le trottoir. Les visites se font essentiellement entre membres de la même famille : «on vit en famille, nous sommes familiers mais on reste dans sa maison, chacun chez soi, les femmes ne sortent pas, on se mélange pas entre gens des quartiers, on se rencontre pour se dire bonjour, pas pour dire de causer », telles sont les expressions employées pour marquer le fait que la famille est le premier cercle de convivialité, le voisinage étant le second. Cependant, la solidarité se met en place dès lors qu'un problème surgit ou que quelqu'un est dans le besoin. Un pêcheur de Grand-Rivière résume bien cela : «à la Lave, tout le monde se connaît, s'il y a un accident, un problème, tout le monde s'entraide, à part ça, chacun reste chez soi ».

Une des raisons invoquées pour justifier cette distance qui s'impose est la peur des milan' : «ça fait des histoires, je n'aime pas m'entremêler des affaires des autres ». Ainsi. ce qui est requis et apprécié dans la vie de voisinage est la discrétion et la distance car l'espace privé est trop limité. Plus on se tient à l'écart des autres et moins, en fait, on donne prise à leur surveillance, à leur jolouzi et, par conséquent, à tout acte de sorcellerie. Toutefois, se tenir trop à distance peut être aussi jugé comme un comportement déviant. Or, celui-ci induit une marginalisation. Ainsi, des personnes distantes ou trop différentes sont traitées « d'aristocrates».

Ces interactions révèlent un ordre, un «ordre normatif [...]. l'ordre comportemental qui existe en tout lieu fréquenté, public, semi-public ou privé que ce soit sous les auspices d'une manifestation sociale élaborée ou sous les contraintes plus banales d'un cadre social routinier» (GOFFMAN. 1974:8).

L'attitude adéquate se situe probablement à mi-chemin entre sympathie et méfiance et consiste à trouver la juste mesure entre la dissimulation et l'ostentation. c'est-à-dire qu'il faut user tantôt dè discrétion, tantôt de provocation afin d'instituer une limite entre soi et les «autres».

Cette frontière entre espace privé-espace public ne peut être que fragile et fluctuante. par C. BOUGerOL, 1997 : 31-51. 
Gestion du risque et de l'oléatoire

por les pêcheurs martiniquais

\section{Les équipages de l'instant}

Dans leur pratique professionnelle, les hommes ont la même stratégie de distanciation. Ce qui caractérise le plus les pêcheurs est effectivement le refus de coercition. de contrainte qui les obligerait à une quelconque pratique collective à long terme, mettant en danger leur choix d'un travail individuel. Le meilleur exemple est l'association au sein des équipages qui repose sur une relation à court terme, sans aucun engagement dans le temps. Les rares équipages qui s'inscrivent dans la durée sont constitués de frères. À terre, l'équipage se disloque, aucun travail de fabrication ou de réparation des agrès de pêche n'est effectué en commun, seul le patron s'attelle à la tâche. La mobilité des matelots est la norme.

II s'avère, en fait, que tout ce qui induirait un contrôle social est rejeté ou détoumé Par exemple, au-delà de l'aspect économique incontournable l'obligation de s'enrôler contrarie bon nombre de pêcheurs car cela les fige dans un statut". C'est une des raisons qui, en dehors de celles d'ordre économique, explique le caractère courant de cette pratique illégale. Les Affaires maritimes ont d'ailleurs été conduites à mettre en place un demi-rôle èt les pêcheurs passent de la légalité à l'illégalité selon leur stratégie (DuBOST, 1996).

Les relations professionnelles sont solides, mais fragmentées, expression d'une atomisation pour préserver l'indépendance de chacun. C'est effectivement parce quill leur laisse une liberté d'action et une indépendance dans l'exercice de leur travail que les pêcheurs déclarent aimer leur métier. Le statut de patron étant le plus valorisé, tout matelot attend le moment adéquat pour se mettre à son compte afin de ne dépendre de personne, de n'être redevable de rien. Cette forme d'individualisme est une garantie pour être soi-même au milieu des autres. afin d'échapper à une proximité trop pesante. Pour un temps, des compétences et une force de travail sont mises en commun, mais l'investissement est dans le court terme et la contrainte doit être la plus légère possible.

\section{Entre contrôle social et atomisation}

Dans un tel univers social, il est important pour l'ordre social de maintenir un espace privé, un consensus, pour que les différends ne se transforment pas en animosité réelle. Cet espace privé est non seulement physique - la maison - mais aussi symbolique, associé à la recherche d'une sphère privée de réalisation. Celle-ci peut être analysée comme un champ de liberté pour un individu ou pour une famille qui garde ainsi une intimité et une indépendance leur permettant de vivre en désamorçant la pression sociale. Mais cela n'est pas élaboré consciemment. c'est de l'ordre du vécu et du non-dit. En aucune façon, il s'agit de nier le collectif. mais tout simplement de se préserver au milieu des «autres», de se prémunir des « autres》.

Le contrôle social se définit comme un système mis en place par un groupe ou une société pour organiser et maintenir lordre social en évitant les pratiques déviantes grâce à des règles. des principes et des valeurs assorties de prescriptions et de sanctions. Le contrôle social qui s'opère dans les villages de pêcheurs illus-

\footnotetext{
"Le róle est une lourde charge financière sur le budget familial annuel. étant de l'ordre c. 2? $000 \mathrm{~F}$ ( $3500 \mathrm{E})$ pour un patron et $24000 \mathrm{~F}$ ( $3658 \mathrm{E})$ pour un matelot.
} 
La pêche oux. Antilles

(Martinique, Guodeloupe)

tre linterdépendance. parfois très contraignante, qui existe dans des espaces de vie relativement limités. II est contrebalancé par une forme atomisée d'organisation professionnelle et sociale avec des échanges restreints, ponctuels et, au demeurant, très efficaces. Les contraintes collectives, dès qu'elles deviennent trop fortes sont rejetées. II s'agit, en fait, de refuser toute forme d'institutionnalisation des rapports sociaux au profit de types d'association et de cohésion sociales limitées dans le temps et dans l'espace. limitées dans la pratique et les effets attendus. Le lien social est puissant mais fragmentaire : face à lomniprésence des «autres », il est renforcé en cas de nécessité, spontanément et efficacement, mais jamais de manière durable.

Je reprends ici les citations de $\mathrm{M}$. Jollivet et $\mathrm{H}$. Mendras dans leur étude sur le monde rural que J. L. BONNIOL prenait déjà comme référence dans son analyse sur les Saintes (1980) : «Tout groupe d'interconnaissance doit aménager une forme d'équilibre entre le quant-à-soi de l'individu et de la famille et la communauté de vie qu'il implique [...].Vivant dans l'intimité des autres, dans une société d'interconnaissance, il est essentiel que chacun marque ses distances et fasse preuve de retenue sans quoi la communauté et la promiscuité seraient complètes et insupportables » (JOLunet et MenDras, 1973: 187-189).

Si un individualisme aussi prégnant est le résultat des forces sociales en jeu, il n'est pas une fin en soi, un but à atteindre. II n'est pas une valeur morale. c'est-à-dire un principe constitutif de sens, déterminant des types de comportements. Bien au contraire, il est interprété comme une caractéristique négative, intrinsèque à la société attachée au principe d'égalité. Les pêcheurs en parlent comme d'une déviance qu'il faut combattre. Chacun sait, s'il veut réussir, quill est seul pour forcer le destin afin d'être meilleur que les « autres » et cela passe par des pratiques individualistes. Certes, la valeur d'égalité peut apparaître comme une compensation à cet individualisme : «cet égalitarisme affirmé n'est souvent qu'une compensation idéologique des différences qui existent en fait dans la position sociale et économique de chaque famille, que chacun connaît et accepte, mais qui doivent être niées, si l'on veut qu'une petite collectivité ait une vie sociale» (JOUVET et Mendras, 1973 : 189). De même, pour PrICE (1964: 100) ce souci d'égalité est «un violent penchant au conformisme social et économique qui compense en quelque sorte une éthique de la concurrence ».

\section{Conclusion}

Dans un contexte historique de fragmentation sociale et de hiérarchie, dont on continue à retrouver tous les stigmates aujourd hui, il semble que ce jeu entre des pratiques holistes et des pratiques individualistes fonctionne comme un verrouillage pour éviter toutes les déviances, alors mềme que le système le permet.

En effet, l'entreprise qui consiste à changer ou à améliorer son statut social, est une nécessité dans cette société construite sur l'esclavage qui nia et bafoua le statut même de lindividu, dans son être, nivelant par là tous les hommes au rang de 
Gestion durisque et de l'aléatoire

par les pecheurs martiniquais

main-d'œuvre. Dès son arrivée sur le sol antillais, l'esclave perdait son nom pour se voir imposer uné dénomination catholique. On lui imposait une abstraction de lui-même. le séparant de sa famille et de son groupe ethnique d'origine, lui interdisant de pratiquer ses rites ou d'exprimer son identité culturelle. II devenait l'autre de lui-même et c'est cette individualité-là quill a fallu reconquérir, par l'élaboration d'un territoire de vie et de sens. Cette problématique demeure comme une sorte de lourdeur qui envahit toute la vie sociale d'autant plus que la métropole. par la départementalisation, continue d'entretenir une situation de dépendance.

L'esclavage et la société de plantation ont entraîné une hiérarchie sociale rigide et tenace malgré les changements intervenus. Celle-ci a engendré au niveau de l'imaginaire sa propre inversion : l'égalité demeure une valeur idéale. Ceperidant, il ne nous semble pas que l'individualisme ait été la réponse à cette situation, mais plutôt le résultat des forces en jeu, sauf peut-être au moment de l'affranchissement des esclaves'". Par contre, selon JOLIVET (1990:55), la petite habitation créole s'est constituée en Guyane grâce à «l'idéologie du contre-pouvoir [qui] y avait joué un rôle moteur plaçant l'individualisme au cour du dispositif» car «l'individualisme marquant le travail et la vie sur l'habitation ou sur les "placers" était avant tout l'expression la plus tangible de la liberté retrouvée», tandis qu'à la Martinique, il «serait à la fois le signe et le moyen de l'ascension sociale ».

Ainsi, instable est léquilibre entre contrôle social et affirmation de l'individu. Les pêcheurs martiniquais ont créé un univers où se mêlent les valeurs de réussite individuelle et d'égalité sociale pour résoudre les conflits et maintenir un consensus garanti par une frontière entre espace public et espace privé. Le risque est essentiellement d'ordre social, il est bien celui de la relation et de l'interaction, et sa gestion conșiste donc à contrebalancer ses effets par des attitudes de méfiance et de distanciation et des pratiques individualistes qui désamorcent tout conflit. Ainsi, au-delà du risque et de l'aléatoire inhérents au métier même de pêcheur et à sa relation à la mer, l'individu est ici confronté à des contraintes sociales particulières qui structurent son univers.

Il apparaît alors évident que les politiques de pêche seront plus efficaces si elles tiennent compte de ces paramètres. Tout projet de développement doit s'inscrire dans l'univers social des pêcheurs pour, d'abord, trouver une légitimité, ensuite être réalisé par les pêcheurs eux-mêmes.

En effet, les pêcheurs revendiquent à travers leurs pratiques techniques et sociales une indépendance qui les positionne comme des acteurs de leur devenir et non comme des assistés. Cette recherche d'autonomie pourrait devenir la pierre angulaire sur laquelle fonder le développement. II se trouve que la cohésion éphémère des équipages. l'observation mutuelle entre professionnels, la recherche d'une excellence dans la pratique. l'ambivalence entre égalité et dépassement des statuts - toujours activée par des pratiques rituelles (DUBOST. 1995) - sont autant d'éléments sur lesquels on peut s'appuyer pour stimuler une dynamique productive. Devenir son propre patron, ne dépéndre de personne. tendre à devenir le meilleur sont des attitudes qui génèrent un esprit d'entreprise, menant à la modernité (DUBOST. 2001).

${ }^{18}$ L'abolition de l'esclavage pour ies Antılles françaises date de 1848 
La péche oux Antilles

(Martinique. Guadeloupe)

L'appropriation et la gestion des DCP par les pêcheurs depuis une dizaine d'années montrent bien leur capacité à innover et à s'adapter à de nouvelles situations, dès lors qu'elles s'inscrivent dans leur contexte social et respectent leurs normes (DuBOst, sous presse). Au contraire, l'échec du contrat État-Région appelé Plan Pêche met en évidence qu'un transfert de techniques et de compétences exogène ne trouve pas de légitimité si les pêcheurs ne se sentent pas concernés. Au-delà des exigences balieutiques et économiques. un développement global et durable ne pourra intervenir que s'il tient compte des attentes des pêcheurs et s'il s'appuie sur leurs fondements sociaux.

Depuis les années 1990, une nouvelle génération de pêcheurs tend à rompre avec la tradition et se montre favorable à un processus de modemisation et de développement de la pêche" ${ }^{\prime \prime}$. Cela correspond aussi à une structuration de la profession avec pour principal acteur le Comité régional des pêches. Cette dynamique sociale dans laquelle s'inscrivent aujourd'hui les pêcheurs ne laisse pas place à l'immobilisme. Bien au contraire, dès lors que leur mode de vie et leurs règles sociales sont préservés, les pêcheurs demeurent ouverts à l'innovation. souhaitant en être les acteurs.

\footnotetext{
${ }^{19}$ En l'espace de quelques années, les pêcheurs ont modifié leurs techniques. se sont adaptés à de nouveaux engins (GPS. DCP...). sont plus sensibles au problème de sécurité et participent activement au développement de leur activité.
} 